\title{
Single-cell analysis reveals melanocytes may promote inflammation in chronic wounds through proteinase-activated receptor-2
}

\section{Aobuliaximu Yakupu}

Department of Burn, Ruijin Hospital, Shanghai Jiao Tong University School of Medicine, Shanghai

\section{Di Zhang}

Department of Burn, Ruijin Hospital, Shanghai Jiao Tong University School of Medicine, Shanghai

\section{Haonan Guan}

Department of Burn, Ruijin Hospital, Shanghai Jiao Tong University School of Medicine, Shanghai

\section{Minfei Jiang}

Department of Burn, Ruijin Hospital, Shanghai Jiao Tong University School of Medicine, Shanghai

\section{Jiaoyun Dong}

Department of Burn, Ruijin Hospital, Shanghai Jiao Tong University School of Medicine, Shanghai

\section{Yiwen Niu}

Department of Burn, Ruijin Hospital, Shanghai Jiao Tong University School of Medicine, Shanghai

Jiajun Tang

Department of Burn, Ruijin Hospital, Shanghai Jiao Tong University School of Medicine, Shanghai

Yingkai Liu

Department of Burn, Ruijin Hospital, Shanghai Jiao Tong University School of Medicine, Shanghai

\section{Xian Ma}

Department of Burn, Ruijin Hospital, Shanghai Jiao Tong University School of Medicine, Shanghai

Shuliang Lu (D 13901738685@139.com )

Department of Burn, Ruijin Hospital, Shanghai Jiao Tong University School of Medicine, Shanghai

\section{Research Article}

Keywords: Melanocytes, Computational biology, Wounds and injuries, Regeneration, Cell communication

Posted Date: February 11th, 2022

DOI: https://doi.org/10.21203/rs.3.rs-1301163/v1

License: (1) (i) This work is licensed under a Creative Commons Attribution 4.0 International License. Read Full License 


\section{Abstract}

\section{Background}

Wound healing is a complex, highly regulated process that is critical for restoring the anatomic and functional integrity of the skin after injury, which requires the interaction of various cell types. During acute wound healing, epidermal cells will activate at precise temporal stages, and proper communication will occur between different cells and will restore the skin integrity eventually. However, why the chronic wounds stagnate in an undefined non-healing state and how the epidermal cells interact with each other in the chronic wound environment is still unclear. To provide new insights into chronic wound healing, we reconstructed the variations in the epidermal cell-cell communication network that occur in chronic wound healing via single-cell RNA-seq (scRNA-seq) data analysis.

\section{Methods}

We used the count matrix of scRNA-seq data to create a Seurat object with the R package "Seurat" which was analyzed using default parameters. GO analysis was performed for the differentially expressed genes with Enrichr by R package "Cluster Profiler". Then we created a CellChat object with the R package "CellChat" from Seurat and reconstructed the cell-cell autocrine and paracrine signaling interactions through CellChatDB.

\section{Results}

Six cell types are annotated in each wound type and through comparing the acute and chronic wounds we found that cellular and molecular interactions of epidermal cells are enhanced in chronic wounds. Especially, the PTN and PAR signaling pathways were significantly changed in chronic wounds. Then we found the melanocyte cell population was the dominant sender of PTN and PAR signaling pathways and the most contributing ligand-receptor pairs of the PTN and PAR signaling pathways were PTN-SDC1 and CTSG-F2RL1 respectively.

\section{Conclusions}

In this study, we used CellChat to reconstruct the epidermal cell-cell autocrine and paracrine signaling networks and try to understand the underlying molecular and physiological perturbations in non-healing wounds. we speculate that melanocytes may play a key role in skin regeneration, and that the absence of the PTN pathway and increasing PAR signaling pathways may disrupt the healing in chronic wounds. In addition, we identified that CTSG and PTN may respectively be a novel potential target and a therapeutic strategy in the treatment of chronic wounds.

\section{Background}

Wound healing is a complex, highly regulated process that is critical for restoring the anatomic and functional integrity of the skin after injury, which requires the interaction of various cell types. There is no 
specific definition of a chronic wound, but it is widely accepted that acute wounds undergo the coherent and overlapped four stages of wound healing, including hemostasis, inflammation, proliferation, and remodeling/maturation.

In contrast, chronic wounds fail to progress normally through these canonic stages, often characterized by a stalled undefined non-healing state, where dysregulated inflammation hinders the regeneration process, causing decreased angiogenesis, hyperproliferative non-migratory epithelium, dysregulated levels of cytokines/growth factors, and/or increased protease activity, and fibrosis [1-5]. In addition, hyperpigmentation of the skin can be found along the edges of most chronic wounds, whereas in some cases hypopigmentation can be found post-recovery. Various pathological states result in the development of chronic lesions, including arterial or venous insufficiency, diabetes, undue pressure on the skin, presence of a foreign body, and infection [6], contributing to high mortality rates and medical costs [7].

The epidermis is an external, stratified epithelium [8] constituted by several distinct cell populations. Among these, keratinocytes and melanocytes are prevalent, but a smaller population of immune cells can also be found. Keratinocytes are arranged in four layers to protect subcutaneous tissues and are in contact with melanocytes, dendritic, neural crest-derived cells that synthesize melanosomes $[9,10]$. Keratinocytes and melanocytes compose "the epidermal melanin unit" anatomical relationship, and it is reckoned that each melanocyte is in contact with around forty keratinocytes in the basal and suprabasal layers, with melanosomes being transported via dendrites to the surrounding keratinocyte [11, 12]. Immune cells participate in skin adaptive and innate immunity, regulate every stage of wound healing via the secretion of various cytokines, chemokines, and growth factors [13-16].

During acute wound healing, epidermal cells will activate at precise temporal stages, and proper communication will occur between resident or recruited, immune or non-immune cells. However, why the chronic wounds stagnate in an undefined non-healing state and how the epidermal cells interact with each other in the chronic wound environment is still unclear. To provide new insights into chronic wound healing, we reconstructed the variations in the epidermal cell-cell communication network that occur in chronic wound healing via single-cell RNA-seq (scRNA-seq) data analysis.

\section{Methods}

\section{scRNA-seq data and analysis workflow}

We retrieved the filtered read count matrix of 1170 cells from uninjured skin and wound epidermis (GSE137897) from the GEO. We next used the count matrix to create a Seurat object with the R package "Seurat" (Version 4.0.4) both for data containing (i.e., the count matrix) and analysis (i.e., Normalizing, PCA, clustering, and UMAP/tSNE); then, the single-cell datasets were analyzed using default parameters[17].

Gene ontology (GO) and Kyoto Encyclopedia of Genes and Genomes (KEGG) analyses 
GO analysis was performed for the differentially expressed genes (DEGs) with Enrichr by R package "Cluster Profiler" (Version 4.0.5) $[18,19]$. Among the $\mathrm{GO}$ biological process terms with $p<=0.01$ and KEGG terms with $\mathrm{p}<=0.05$.

\section{Inference and analysis of cell-cell communication}

We created a CellChat object with the R package "CellChat" (Version 1.1.3) from Seurat and reconstructed the cell-cell autocrine and paracrine signaling interactions through CellChatDB, a manually curated database, to identify the conserved and context-specific signaling pathways in different wound healing conditions[20].

\section{Results}

\section{Classification of epidermal cell constitution of uninjured skin and wounds}

We retrieved and analyzed the filtered read count matrix of 1170 cells from three different conditions, i.e., normal skin $(n=391)$, acute wounds (AW, $n=398)$, and pressure ulcers ( $P U, n=381)$ (cells that passed the quality control) from the GEO(GSE137897) [21]. These epidermal cells were separated into six clusters each by Seurat clustering (Figure 1A, B, C). The annotation based on that basal layer keratinocyte had the highest expression levels of KRT5 and KRT14; spinous keratinocyte displayed high DSG1 and DSP levels with the highest expression of KRT1 and KRT10; granular keratinocyte expressed a suite of late differentiation markers, including LOR, FLG, and SPINK5; and melanocytes had a higher expression of PMEL, TYRP1, and MLANA, whereas immune cells had higher levels of CD74. Combining canonical, novel markers in differential analysis and KEGG/GO analysis allowed us to annotate different clusters (Figure $1 D, E, F, G)[22-26]$.

In uninjured skin, we annotated one basal $(n=106)$, granular $(n=74)$, and spinous $(n=74)$ keratinocyte cluster, one cluster named melanocytes $(n=65)$, one for immune cell $(n=58)$ and one termed as "mitotic" $(n=14)$ because of the high level of well-recognized DNA synthesis and cell division transcripts, such as PCNA and KI67 (Figure 1H).

Following the same criterion, we annotated two basal $(n=88$ and $n=72)$ keratinocyte clusters, one spinous $(n=100)$ keratinocyte cluster, one melanocytes cluster $(n=11)$, one immune cell cluster $(n=85)$ and one "mitotic" cluster $(n=42)$ in AW (Figure 1I). In PU, we annotated one basal $(n=66)$ and one spinous $(n=66)$ keratinocyte cluster respectively, one melanocyte $(n=31)$ cluster, two immune cell clusters $(n=83$ and $n=81)$ and a "mitotic" cluster $(n=54)$ (Figure $1 \mathrm{~J})$.

\section{Cellular and molecular interactions of epidermal cells are enhanced in chronic wounds}

We used CellChat to predict the general principles of cell-cell communication. Comparing the total number of interactions and interaction strength, we found that cell-cell communication was enhanced in chronic wound conditions (Figure 2A). Furthermore, we compared the number of interactions and interaction strength among different cell populations to identify those which showed significant changes. 
We found that signaling was increased between immune cells and other cells in PU compared with AW. Spinous and mitotic cell population communication was increased in PU, where mitotic cell populations were the busiest in signaling. The interaction between mitotic cells and immune cells was strongest in PU (Figure 2B, C, D).

\section{PTN and PAR signaling pathways were significantly changed in chronic wounds}

We identified the significantly altered signaling pathways by simply comparing the information flow for each, which is defined by the sum of communication probabilities among all pairs of cell groups in the inferred network (i.e., the total weights in the network). Significantly changed signaling pathways were ranked based on differences in the overall information flow within the inferred networks between the AW and PU. As shown in Figure 3A, the leading signaling pathways depicted in red are enriched in AW, whereas those in green are enriched in PU. The results show the signaling pathways with the strongest variation in increase or decrease of activity, which include those of pleiotrophin (PTN), prosaposin (PSAP), Secreted Ly-6/uPAR-related protein (SLURP), epidermal growth factor (EGF), VISVATIN, which also called visfatin, calcitonin receptor (CALCR), and the protease-activated receptor (PAR) signaling pathway.

We further analyzed the outgoing and incoming signaling associated with each cell population between AW and PU (Figure 3B, C, D). In PU, results showed a major decrease in signaling from melanocytes, such as the PTN and PSAP pathways, whereas the highest increase was registered in the PAR pathway, also from the melanocyte population. Other significantly changed signaling pathways were EGF, visfatin, and CALCR from the basal cell population. Overall, signaling pathways from the melanocyte population were the most altered between AW and PU. This indicates that melanocytes may play an important role in wound healing.

\section{The melanocyte cell population was the dominant sender in PTN and PAR signaling pathways}

To define the signaling sources most subject to variation, we identified the dominant senders, receivers, mediators, and influencers in the substantially altered pathways. The PTN signaling pathway was only present in AW, where melanocytes are the senders, and spinous, basal, immune, and mitotic cells are the receivers. The major mediators and influencers of the PTN signaling pathway are the immune cells (Figure 4A). In the PAR signaling pathway, the major senders are the melanocytes, whereas the spinous and mitotic cells are the main receivers and accept almost all the other cells' influence (Figure 4B). Therefore, the absence of the PTN signaling pathways and the increased PAR signaling pathway from the melanocytes of chronic wounds, indicate that melanocytes may play a crucial role in the healing process.

Next, we identified the upregulated and downregulated signaling ligand-receptor pairs in PU and determined the communication probabilities mediated by ligand-receptor pairs from melanocytes to other cell groups (Figure 4C). This showed that the highest communication probabilities mediated by ligandreceptor pairs are substantially changed from melanocytes compared to other cell groups, such as CTSG- 
F2RL1, CTSG-PARD3, CTSG-F2R, which belong to the PAR signaling pathway, and PTN-SDC1, PTN-SDC3, and PTN-NCL, which are part of the PTN signaling pathway.

\section{PTN-SDC1 pairs in the PTN signaling pathway may regulate epithelial cell proliferation and migration during wound healing}

To determine what type of effects the substantially changed signaling pathways may bring to the receiver cell populations, we calculated the most contributing ligand-receptor pairs in cell-cell communication in the most altered signaling pathway. We narrowed down the analysis to the most highly contributing pairs to analyze the information between sender and receiver cells.

The most contributing ligand-receptor pair of the PTN signaling pathway, which is only present in AW, was PTN-SDC1 (Figure 5A, B). PTN is only expressed in the melanocyte population and is a secreted growth factor that mediates signaling via cell-surface proteoglycan and non-proteoglycan receptors via their chondroitin sulfate (CS) groups [27-31]. Syndecan-1 (SDC1) is a cell surface proteoglycan that participates in cell-cell and cell-matrix interactions, a growth factor coreceptor involved in wound healing [32-35]. SDC1 expression was the highest in almost all cells, indicating that melanocytes can affect other cell populations through PTN-SDC1 binding (Figure 5C, D).

PTN gene expression is upregulated in brain injury [36], and is involved in bone repair [37], angiogenesis [38-40], regulation of epithelial cell migration, and keratinocyte proliferation [41, 42]. In previous studies, PTN and SDC1 expression was found to be upregulated in brain injury and promote epithelial cell proliferation and migration during wound healing. However, the PTN-SDC1 ligand-receptor pair, in other words, the PTN pathway, is absent in PU, so we speculate that melanocytes may play a key role in skin regeneration, and that the absence of the PTN pathway may disrupt healing in chronic wounds.

\section{CTSG-F2RL1 pairs in the PAR signaling pathway may lead to chronic inflammation in chronic wounds}

PAR signaling pathways are only present in PU. PARs are a family of G-protein-coupled receptors that are irreversibly activated by proteolytic cleavage of their N-terminus. This exposes a peptidic ligand that binds and activates the transmembrane receptor domain, provoking a cellular cascade in response to inflammatory signals and other stimuli. PARs are involved in a wide variety of diseases, such as cancer and inflammation $[43,44]$.

The ligand-receptor pair in the PAR pathway that contributes most to cell-cell communication is CTSGF2RL1 (Figure 6A, B). CTSG, also known as cathepsin G, has a serine protease with trypsin- and chymotrypsin-like specificity. CTSG is synthesized and secreted by neutrophils, mast cells, and antigenpresenting cells [45-48]. CTSG is also found in some non-immune cells, such as endothelial and smooth muscle cells [49], brain astrocytes [50], fibroblasts [51], and in Paneth cells - specialized epithelial cells underneath the crypts of Lieberkühn $[52,53]$. We also found that CTSG was expressed in the melanocyte population of PU. F2RL1 is a receptor for trypsin and trypsin-like enzymes coupled to G proteins and is activated through proteolytic cleavage of its extracellular amino terminus [43]. F2RL1 is expressed in 
almost all cells except melanocytes, and we can deduce that melanocytes can send their signal to other cell populations through CTSG-F2RL1 binding (Figure 6C, D).

Previous studies have found that CTSG is elevated in chronic wounds and inhibits regeneration [54-56] and promotes inflammation [57]. F2RL1 can reduce cell migration through actin cabling and can prevent the internalization of E-cadherin [58]. Its stimulation can promote inflammation and inflammatory cell infiltration [59-61]. Therefore, CTSG binding to F2RL1 may promote inflammation in and reduce migration of the epithelium cells during wound healing. However, CTSG-F2RL1 ligand-receptor pair, i.e., the PAR pathway, is increased in PU, adding evidence that melanocytes may play a key role in wound healing. The increase in the PAR pathway may contribute to chronic inflammation and inhibition of cell migration in the epithelium of chronic wounds.

\section{Discussion}

PU are chronic lesions, localized injuries to the skin and underlying soft tissue caused by pressure, shear, friction, or a combination of these, often present in patients with limited mobility or sensory perception and paralyzed or unconscious patients who can neither sense nor reply to the intermittent need for changing the position[62-65].

The role of melanocytes in wound healing was first observed in the 1950s when it was reported that melanocytes appeared in the new epithelial covering within 4-6 days following the lesion [66]. Although, melanocytes were found to undergo mitosis in the adjacent uninjured skin and synthesize melanin, the melanocytes within the migrating epidermis did not have high melanin expression [67]. Previous studies reported that melanocytes are involved in regulating keratinocyte differentiation [68], which in turn regulate melanogenesis by paracrine effect [69].

To further understand the role of melanocytes in wound healing, one must focus on the intricate cellular and molecular interactions between melanocytes, keratinocytes, and immune cells. We used CellChat to reconstruct the epidermal cell-cell autocrine and paracrine signaling networks and compared the number of interactions and their strength among different cell populations to identify substantial alterations in the interactions between them. Furthermore, we identified which pathways were significantly upregulated or downregulated in PU by comparing the information flow and determined their corresponding signaling ligand-receptor pairs. To identify the main sources in the substantially altered signaling pathway, we identified its dominant senders, receivers, mediators, and influencers. The results showed that the main sources of the most upregulated and downregulated signaling pathways were melanocytes. This suggests that melanocytes may play a crucial role in healing and that these alterations are responsible for hindering the regeneration process in chronic wounds.

To determine how melanocytes affect other cells' behavior by autocrine and paracrine signaling, we calculated the highest contributing ligand-receptor pairs of the substantially altered pathway and found the pairs' gene expression in each cell. We found that melanocytes increase keratinocyte proliferation and migration during skin regeneration mostly via the PTN-SDC1 ligand-receptor pairs of the PTN signaling 
pathway. Previous studies reported that the PTN signaling pathway regulates cell survival, cell growth, cell differentiation in several tissues, namely neuron, and bone, and increases the phosphorylation of AFAP1L2 to activate the PI3K-AKT pathway by binding to PTPRZ1 to regulate oligodendrocyte precursor cell differentiation $[35,70-73]$. However, the PTN signaling pathway is absent in chronic wounds, which may contribute to delayed re-epithelialization.

Additionally, we found that melanocytes may contribute to chronic inflammation and inhibition of cell migration in the epithelium of chronic wounds through CTSG-F2RL1 ligand-receptor pairs. Previous studies reported that CTSG is involved in connective tissue remodeling at sites of inflammation and in the killing and digestion of engulfed pathogens, exerting an antimicrobial function [74-77]. CTSG also binds to PARD3, which increases cell division and cell polarization, increases epithelial tight junctions, and decreases epithelial-to-mesenchymal transition $[78,79]$, which may lead to decreased angiogenesis and hyperproliferative non-migratory epithelium. Although PRSS3-F2R ligand-receptor pairs have low contribution in the PAR signaling pathway, PRSS3 is also a member of the trypsin family of serine proteases [80] and F2R is a member of the PARs, therefore PRSS3-F2R binding may produce a similar effect to CTSG-F2RL1 binding. Melanocytes may regulate other epithelial cells to modulate inflammatory responses or promote inflammation via the PAR signaling pathway, which is upregulated in PU and may result in chronic inflammation in chronic wounds. Overall, skin lesion stimulates the proliferation of melanocytes in wound-adjacent uninjured skin, and melanocytes, in turn, regulate inflammation, cell proliferation, and cell migration during wound healing.

\section{Conclusions}

In this study, we used CellChat to reconstruct the epidermal cell-cell autocrine and paracrine signaling networks and try to understand the underlying molecular and physiological perturbations in non-healing wounds. The PTN-SDC1 signaling pair, from the PTN signaling pathway, may promote epithelial cell proliferation and migration in wound healing; this depends on melanocytes and is absent in chronic wounds. The CTSG-F2RL1 signaling pair in the PAR pathway, in contrast, increases inflammation and reduces epithelial cell migration during healing; in PU, the signals of this pathway that originate from melanocytes are upregulated, which may lead to a prolonged and increased inflammatory state and inhibition of cell migration in chronic wounds. In conclusion, we identified that CTSG and PTN may respectively be a novel potential target and a therapeutic strategy in the treatment of chronic wounds.

\section{Declarations}

\section{Ethics approval and consent to participate}

All methods were performed in accordance with the relevant guidelines and regulations.

\section{Consent for publication}

Not applicable 
Availability of data and materials

The datasets generated and/or analyzed during the current study are available in the GEO repository (GSE137897), https://www.ncbi.nlm.nih.gov/geo/query/acc.cgi?acc=GSE137897.

\section{Competing interests}

The authors declare that they have no competing interests

\section{Funding}

National Natural Science Foundation of China, Grant/Award Numbers: 81671916.

\section{Authors' contributions}

AY, DZ analyzed and visualized the data. AY, XM, SLL was a major contributor in writing the manuscript. All authors read and approved the final manuscript.

\section{Acknowledgements}

We thank the countless individuals who have contributed to the GEO database in various capacities.

\section{References}

1. Frykberg, R.G. and J. Banks, Challenges in the Treatment of Chronic Wounds. Adv Wound Care (New Rochelle), 2015. 4(9): p. 560-582.

2. Las Heras, K., et al., Chronic wounds: Current status, available strategies and emerging therapeutic solutions. Journal of controlled release : official journal of the Controlled Release Society, 2020. 328: p. 532-550.

3. Martin, P. and R. Nunan, Cellular and molecular mechanisms of repair in acute and chronic wound healing. Br J Dermatol, 2015. 173(2): p. 370-8.

4. Atkin, L., Chronic wounds: the challenges of appropriate management. Br J Community Nurs, 2019. 24(Sup9): p. S26-s32.

5. Sawaya, A.P., et al., Deregulated immune cell recruitment orchestrated by FOXM1 impairs human diabetic wound healing. Nature communications, 2020. 11(1): p. 4678.

6. Jones, R.E., D.S. Foster, and M.T. Longaker, Management of Chronic Wounds-2018. Jama, 2018. 320(14): p. 1481-1482.

7. Sen, C.K., Human Wounds and Its Burden: An Updated Compendium of Estimates. Advances in Wound Care, 2019. 8(2): p. 39-48.

8. Tobin, D.J., Biochemistry of human skin-our brain on the outside. Chemical Society reviews, 2006. 35(1): p. 52-67.

9. Rodrigues, M., et al., Wound Healing: A Cellular Perspective. Physiol Rev, 2019. 99(1): p. 665-706. 
10. Hearing, V.J. and W. Hori, The regulation ofmelanin production. 1997. 3.1.21.

11. Fitzpatrick, T.B. and A.S. Breathnach, [THE EPIDERMAL MELANIN UNIT SYSTEM]. Dermatologische Wochenschrift, 1963. 147: p. 481-489.

12. Costin, G.-E. and V.J. Hearing, Human skin pigmentation: melanocytes modulate skin color in response to stress. The FASEB Journal, 2007. 21(4): p. 976-994.

13. Gurtner, G.C., et al., Wound repair and regeneration. Nature, 2008. 453(7193): p. 314-21.

14. Lowes, M.A., M. Suárez-Fariñas, and J.G. Krueger, Immunology of psoriasis. Annu Rev Immunol, 2014. 32: p. 227-55.

15. Pasparakis, M., I. Haase, and F.O. Nestle, Mechanisms regulating skin immunity and inflammation. Nat Rev Immunol, 2014. 14(5): p. 289-301.

16. Kabashima, K., et al., The immunological anatomy of the skin. Nat Rev Immunol, 2019. 19(1): p. 1930 .

17. Hao, Y., et al., Integrated analysis of multimodal single-cell data. Cell, 2021. 184(13): p. 35733587.e29.

18. Chen, E.Y., et al., Enrichr: interactive and collaborative HTML 5 gene list enrichment analysis tool. BMC bioinformatics, 2013. 14: p. 128.

19. Kuleshov, M.V., et al., Enrichr: a comprehensive gene set enrichment analysis web server 2016 update. Nucleic acids research, 2016. 44(W1): p. W90-W97.

20. Jin, S., et al., Inference and analysis of cell-cell communication using CellChat. Nature communications, 2021. 12(1): p. 1088.

21. Li, D., et al., Single-cell analysis reveals MHCIl expressing keratinocytes in pressure ulcers with worse healing outcomes. bioRxiv, 2021: p. 2021.04.20.440591.

22. Finnegan, A., et al., Single-Cell Transcriptomics Reveals Spatial and Temporal Turnover of Keratinocyte Differentiation Regulators. Frontiers in genetics, 2019. 10: p. 775.

23. Sanz-Gómez, N., A. Freije, and A. Gandarillas, Keratinocyte Differentiation by Flow Cytometry. Methods in molecular biology (Clifton, N.J.), 2020. 2109: p. 83-92.

24. Wang, S., et al., Single cell transcriptomics of human epidermis identifies basal stem cell transition states. Nature communications, 2020. 11(1): p. 4239.

25. Enzo, E., et al., Single-keratinocyte transcriptomic analyses identify different clonal types and proliferative potential mediated by FOXM1 in human epidermal stem cells. Nature communications, 2021. 12(1): p. 2505.

26. Cheng, J.B., et al., Transcriptional Programming of Normal and Inflamed Human Epidermis at SingleCell Resolution. Cell reports, 2018. 25(4): p. 871-883.

27. Stoica, G.E., et al., Identification of anaplastic lymphoma kinase as a receptor for the growth factor pleiotrophin. The Journal of biological chemistry, 2001. 276(20): p. 16772-16779.

28. Fukada, M., et al., Protein tyrosine phosphatase receptor type $Z$ is inactivated by ligand-induced oligomerization. FEBS letters, 2006. 580(17): p. 4051-4056. 
29. Mikelis, C., et al., Integrin alpha(v)beta(3) is a pleiotrophin receptor required for pleiotrophin-induced endothelial cell migration through receptor protein tyrosine phosphatase beta/zeta. FASEB journal : official publication of the Federation of American Societies for Experimental Biology, 2009. 23(5): $p$. 1459-1469.

30. Kuboyama, K., et al., Role of Chondroitin Sulfate (CS) Modification in the Regulation of Proteintyrosine Phosphatase Receptor Type Z (PTPRZ) Activity: PLEIOTROPHIN-PTPRZ-A SIGNALING IS INVOLVED IN OLIGODENDROCYTE DIFFERENTIATION. The Journal of biological chemistry, 2016. 291(35): p. 18117-18128.

31. Ryan, E., D. Shen, and X. Wang, Structural studies reveal an important role for the pleiotrophin Cterminus in mediating interactions with chondroitin sulfate. The FEBS journal, 2016. 283(8): p. 14881503.

32. Dews, I.C. and K.R. Mackenzie, Transmembrane domains of the syndecan family of growth factor coreceptors display a hierarchy of homotypic and heterotypic interactions. Proceedings of the National Academy of Sciences of the United States of America, 2007. 104(52): p. 20782-20787.

33. Baietti, M.F., et al., Syndecan-syntenin-ALIX regulates the biogenesis of exosomes. Nature cell biology, 2012. 14(7): p. 677-685.

34. Elenius, K., et al., Induced expression of syndecan in healing wounds. The Journal of cell biology, 1991. 114(3): p. 585-595.

35. Galko, M.J. and M.A. Krasnow, Cellular and genetic analysis of wound healing in Drosophila larvae. PLoS biology, 2004. 2(8): p. E239.

36. Yeh, H.J., et al., Upregulation of pleiotrophin gene expression in developing microvasculature, macrophages, and astrocytes after acute ischemic brain injury. The Journal of neuroscience : the official journal of the Society for Neuroscience, 1998. 18(10): p. 3699-3707.

37. Li, G., et al., Effects of pleiotrophin (PTN) over-expression on mouse long bone development, fracture healing and bone repair. Calcified tissue international, 2005. 76(4): p. 299-306.

38. Petersen, W., et al., The angiogenic peptide pleiotrophin (PTN/HB-GAM) is expressed in fracture healing: an immunohistochemical study in rats. Archives of orthopaedic and trauma surgery, 2004. 124(9): p. 603-607.

39. Christman, K.L., et al., Pleiotrophin induces formation of functional neovasculature in vivo. Biochemical and biophysical research communications, 2005. 332(4): p. 1146-1152.

40. Weiss, S., et al., The systemic angiogenic response during bone healing. Archives of orthopaedic and trauma surgery, 2009. 129(7): p. 989-997.

41. Silver, K., et al., Expression of pleiotrophin, an important regulator of cell migration, is inhibited in intestinal epithelial cells by treatment with non-steroidal anti-inflammatory drugs. Growth factors (Chur, Switzerland), 2012. 30(4): p. 258-266.

42. Florin, L., et al., Increased keratinocyte proliferation by JUN-dependent expression of PTN and SDF-1 in fibroblasts. Journal of cell science, 2005. 118(Pt 9): p. 1981-1989. 
43. Cheng, R.K.Y., et al., Structural insight into allosteric modulation of protease-activated receptor 2. Nature, 2017. 545(7652): p. 112-115.

44. Shpacovitch, V., et al., Protease-activated receptors: novel PARtners in innate immunity. Trends in immunology, 2007. 28(12): p. 541-550.

45. Schechter, N.M., et al., Determination of the primary structures of human skin chymase and cathepsin $\mathrm{G}$ from cutaneous mast cells of urticaria pigmentosa lesions. Journal of immunology (Baltimore, Md. : 1950), 1994. 152(8): p. 4062-4069.

46. Korkmaz, B., T. Moreau, and F. Gauthier, Neutrophil elastase, proteinase 3 and cathepsin G: physicochemical properties, activity and physiopathological functions. Biochimie, 2008. 90(2): p. 227-242.

47. Stoeckle, C., et al., Cathepsin $\mathrm{G}$ is differentially expressed in primary human antigen-presenting cells. Cellular immunology, 2009. 255(1-2): p. 41-45.

48. Burster, T., et al., Cathepsin G: roles in antigen presentation and beyond. Molecular immunology, 2010. 47(4): p. 658-665.

49. Wang, J., et al., Cathepsin $G$ activity lowers plasma $L D L$ and reduces atherosclerosis. Biochimica et biophysica acta, 2014. 1842(11): p. 2174-2183.

50. Abraham, C.R., K. Kanemaru, and L. Mucke, Expression of cathepsin G-like and alpha 1antichymotrypsin-like proteins in reactive astrocytes. Brain research, 1993. 621(2): p. 222-232.

51. Cavarra, E., et al., UVA light stimulates the production of cathepsin $G$ and elastase-like enzymes by dermal fibroblasts: a possible contribution to the remodeling of elastotic areas in sun-damaged skin. Biological chemistry, 2002. 383(1): p. 199-206.

52. Zamolodchikova, T.S., et al., Cathepsin $G$ in the immune defense of the human duodenum: New sources for biosynthesis. Human Physiology, 2017. 43(3): p. 326-333.

53. Zamolodchikova, T.S., S.M. Tolpygo, and E.V. Svirshchevskaya, Cathepsin G-Not Only Inflammation: The Immune Protease Can Regulate Normal Physiological Processes. Frontiers in immunology, 2020. 11: p. 411.

54. Yager, D.R. and B.C. Nwomeh, The proteolytic environment of chronic wounds. Wound repair and regeneration : official publication of the Wound Healing Society [and] the European Tissue Repair Society, 1999. 7(6): p. 433-441.

55. Greener, B., et al., Proteases and pH in chronic wounds. Journal of wound care, 2005. 14(2): p. 59-61.

56. Edwards, J.V., et al., Protease inhibition by oleic acid transfer from chronic wound dressings to albumin. International journal of pharmaceutics, 2007. 340(1-2): p. 42-51.

57. Liu, X., et al., Up-regulation of Cathepsin G in the Development of Chronic Postsurgical Pain: An Experimental and Clinical Genetic Study. Anesthesiology, 2015. 123(4): p. 838-850.

58. Fernando, E.H., et al., Inhibition of Intestinal Epithelial Wound Healing through Protease-Activated Receptor-2 Activation in Caco2 Cells. The Journal of pharmacology and experimental therapeutics, 2018. 367(2): p. 382-392. 
59. Miike, S., A.S. McWilliam, and H. Kita, Trypsin induces activation and inflammatory mediator release from human eosinophils through protease-activated receptor-2. Journal of immunology (Baltimore, Md. : 1950), 2001. 167(11): p. 6615-6622.

60. Shpacovitch, V.M., et al., Agonists of proteinase-activated receptor-2 modulate human neutrophil cytokine secretion, expression of cell adhesion molecules, and migration within 3-D collagen lattices. Journal of leukocyte biology, 2004. 76(2): p. 388-398.

61. Gao, S., et al., The role and mechanism of cathepsin $G$ in dermatomyositis. Biomedicine \& pharmacotherapy $=$ Biomedecine $\&$ pharmacotherapie, 2017. 94: p. 697-704.

62. Dam, A., et al., Managing pressures ulcers in a resource constrained situation: a holistic approach. Indian journal of palliative care, 2011. 17(3): p. 255-259.

63. Zhao, R., et al., Inflammation in Chronic Wounds. Int J Mol Sci, 2016. 17(12).

64. Mervis, J.S. and T.J. Phillips, Pressure ulcers: Pathophysiology, epidemiology, risk factors, and presentation. J Am Acad Dermatol, 2019. 81(4): p. 881-890.

65. Zhao, J.-C., et al., Couple-kissing flaps for successful repair of severe sacral pressure ulcers in frail elderly patients. BMC geriatrics, 2017. 17(1): p. 285-285.

66. Snell, R.S., A study of the melanocytes and melanin in a healing deep wound. Journal of anatomy, 1963. 97: p. 243-253.

67. Hirobe, T., Proliferation of epidermal melanocytes during the healing of skin wounds in newborn mice. Journal of Experimental Zoology, 1983. 227(3): p. 423-431.

68. Paus, R., Migrating melanocyte stem cells: masters of disaster? Nature Medicine, 2013. 19(7): p. 818819.

69. Yuan, X.H. and Z.H. Jin, Paracrine regulation of melanogenesis. The British journal of dermatology, 2018. 178(3): p. 632-639.

70. Wellstein, A., et al., A heparin-binding growth factor secreted from breast cancer cells homologous to a developmentally regulated cytokine. The Journal of biological chemistry, 1992. 267(4): p. 25822587.

71. Kretschmer, P.J., et al., Cloning, characterization and developmental regulation of two members of a novel human gene family of neurite outgrowth-promoting proteins. Growth factors (Chur, Switzerland), 1991. 5(2).

72. Tanga, N., et al., The PTN-PTPRZ signal activates the AFAP1L2-dependent PI3K-AKT pathway for oligodendrocyte differentiation: Targeted inactivation of PTPRZ activity in mice. Glia, 2019. 67(5): p. 967-984.

73. Imai, S., et al., Osteocyte-derived HB-GAM (pleiotrophin) is associated with bone formation and mechanical loading. Bone, 2009. 44(5): p. 785-794.

74. Avril, L.E., et al., Identification of the U-937 membrane-associated proteinase interacting with the V3 loop of HIV-1 gp120 as cathepsin G. FEBS letters, 1994. 345(1): p. 81-86. 
75. Wasiluk, K.R., K.M. Skubitz, and B.H. Gray, Comparison of granule proteins from human polymorphonuclear leukocytes which are bactericidal toward Pseudomonas aeruginosa. Infection and immunity, 1991. 59(11): p. 4193-4200.

76. Maison, C.M., C.L. Villiers, and M.G. Colomb, Proteolysis of C3 on U937 cell plasma membranes. Purification of cathepsin G. Journal of immunology (Baltimore, Md. : 1950), 1991. 147(3): p. 921926.

77. Ferrell, W.R., et al., Essential role for proteinase-activated receptor-2 in arthritis. The Journal of clinical investigation, 2003. 111(1): p. 35-41.

78. Chen, $\mathrm{X}$., et al., Rare Deleterious PARD3 Variants in the aPKC-Binding Region are Implicated in the Pathogenesis of Human Cranial Neural Tube Defects Via Disrupting Apical Tight Junction Formation. Human mutation, 2017. 38(4): p. 378-389.

79. Johansson, A., M. Driessens, and P. Aspenström, The mammalian homologue of the Caenorhabditis elegans polarity protein PAR-6 is a binding partner for the Rho GTPases Cdc42 and Rac1. Journal of cell science, 2000. 113 (Pt 18): p. 3267-3275.

80. Szmola, R., Z. Kukor, and M. Sahin-Tóth, Human mesotrypsin is a unique digestive protease specialized for the degradation of trypsin inhibitors. The Journal of biological chemistry, 2003. 278(49): p. 48580-48589.

\section{Figures}



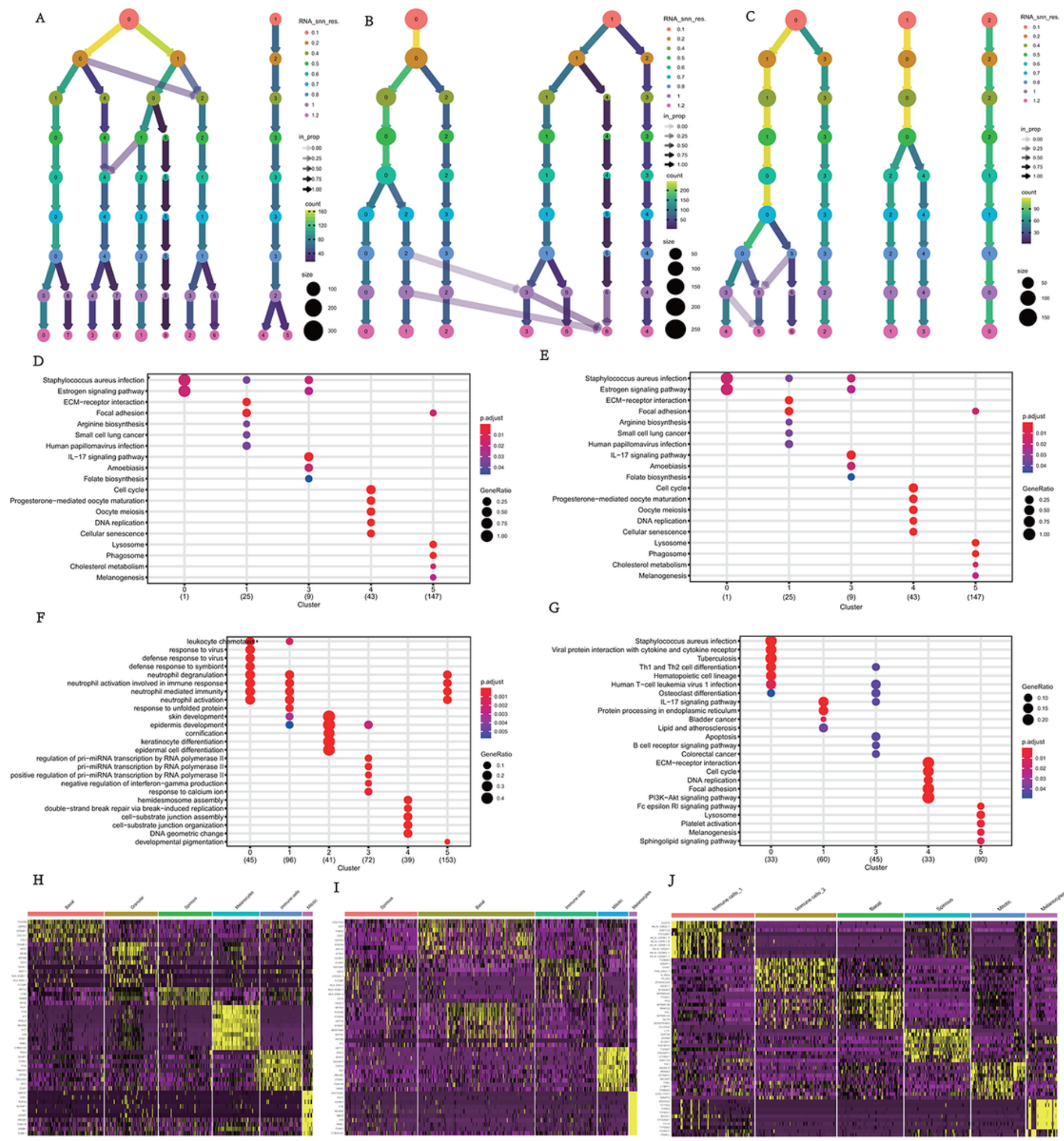

$\mathrm{J}$

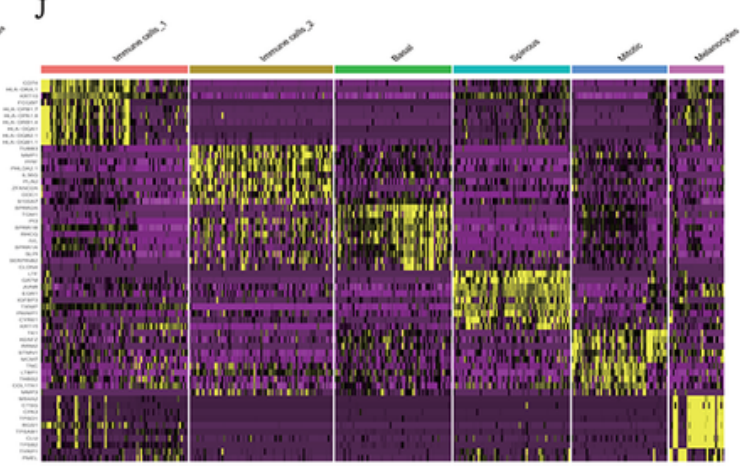

Figure 1

Classification of uninjured skin and wound epidermal cell constitution. A, B, C, Cluster number of different resolutions in three sample types. D, E, F, G, GO and KEGG analyses of each cluster for cluster annotation. H, I, J, Heatmap of one cell population's top 10 genes' expression to other cell populations(yellow), some genes are not shown. 
A

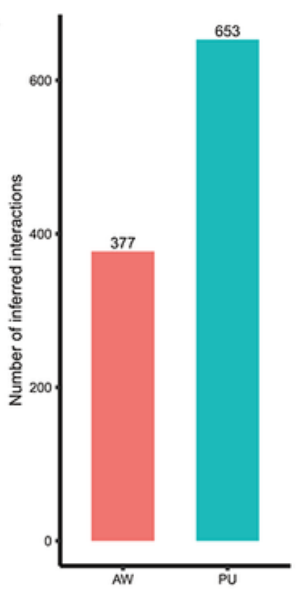

C

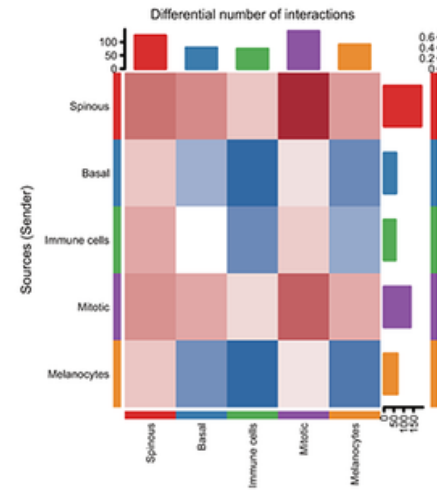

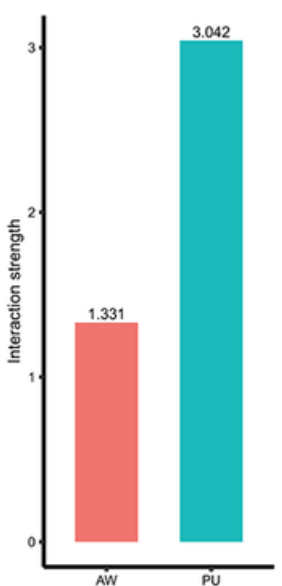

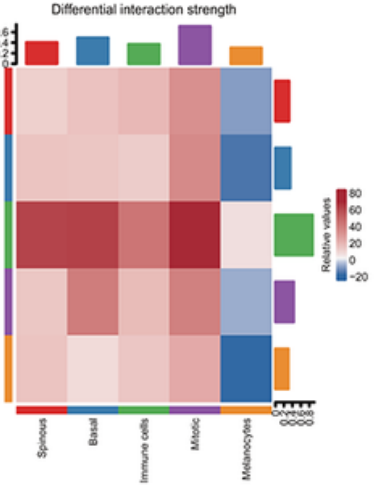

B

$\mathrm{D}$
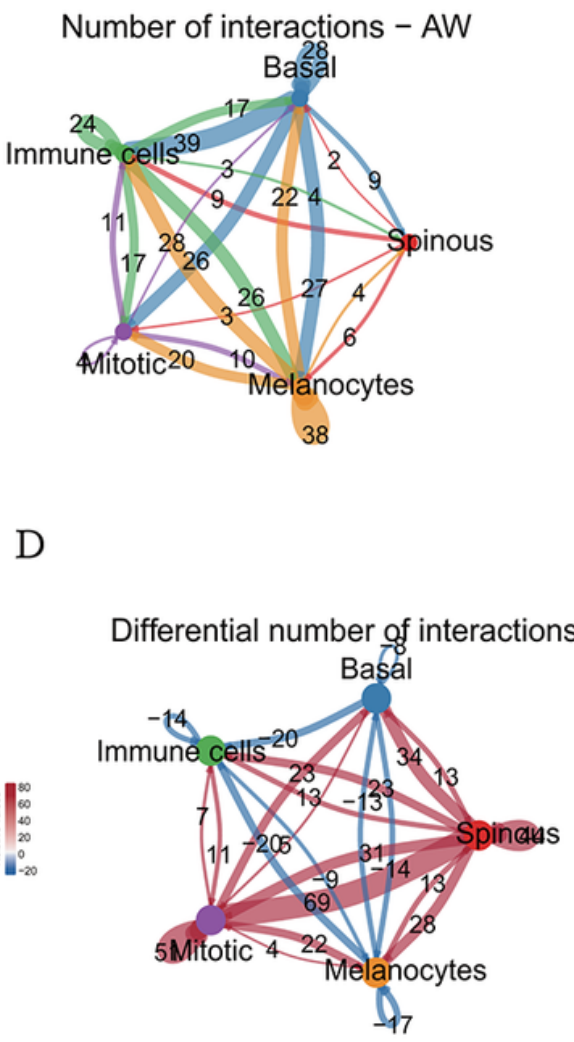
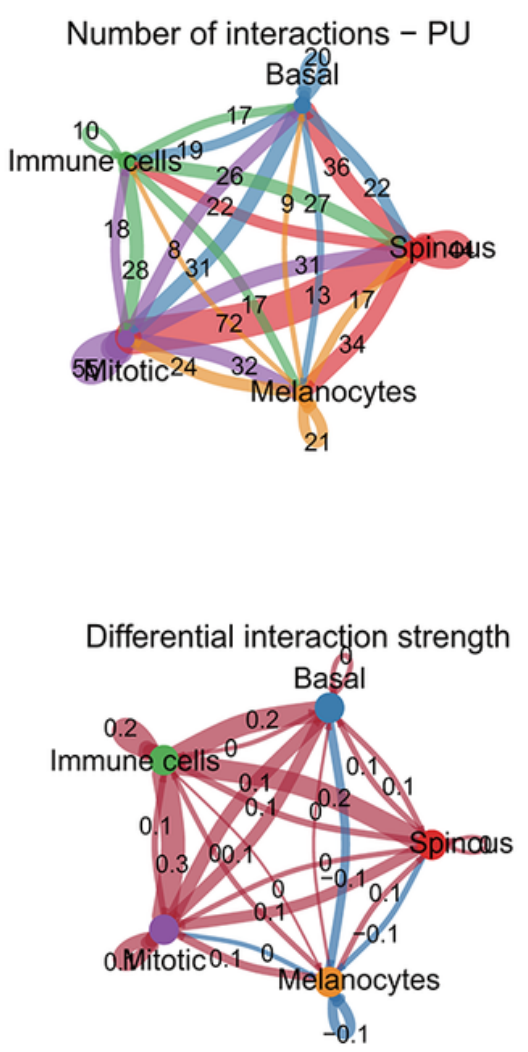

Figure 2

Comparison of the number of interactions and interaction strength among different cell populations. A, Comparison of the total number of interactions and interaction strength; cell-cell communication is enhanced in chronic wounds. B, The number of interactions between any two cell populations in acute wounds (AW) and pressure ulcers (PU). C, The differential number of interactions or interaction strength in the cell-cell communication network between AW and PU. The top colored bar plot represents the sum of the column of values displayed in the heatmap (incoming signals). The right colored bar plot represents the sum of the row of values (outgoing signals). In the color bar, red (or blue) represents increased (or decreased) signaling in the PU compared to AW. D, The differential number of interactions and interaction strength between any two cell types. Red (or blue) colored edges represent increased (or decreased) signaling in the PU compared to the AW. 

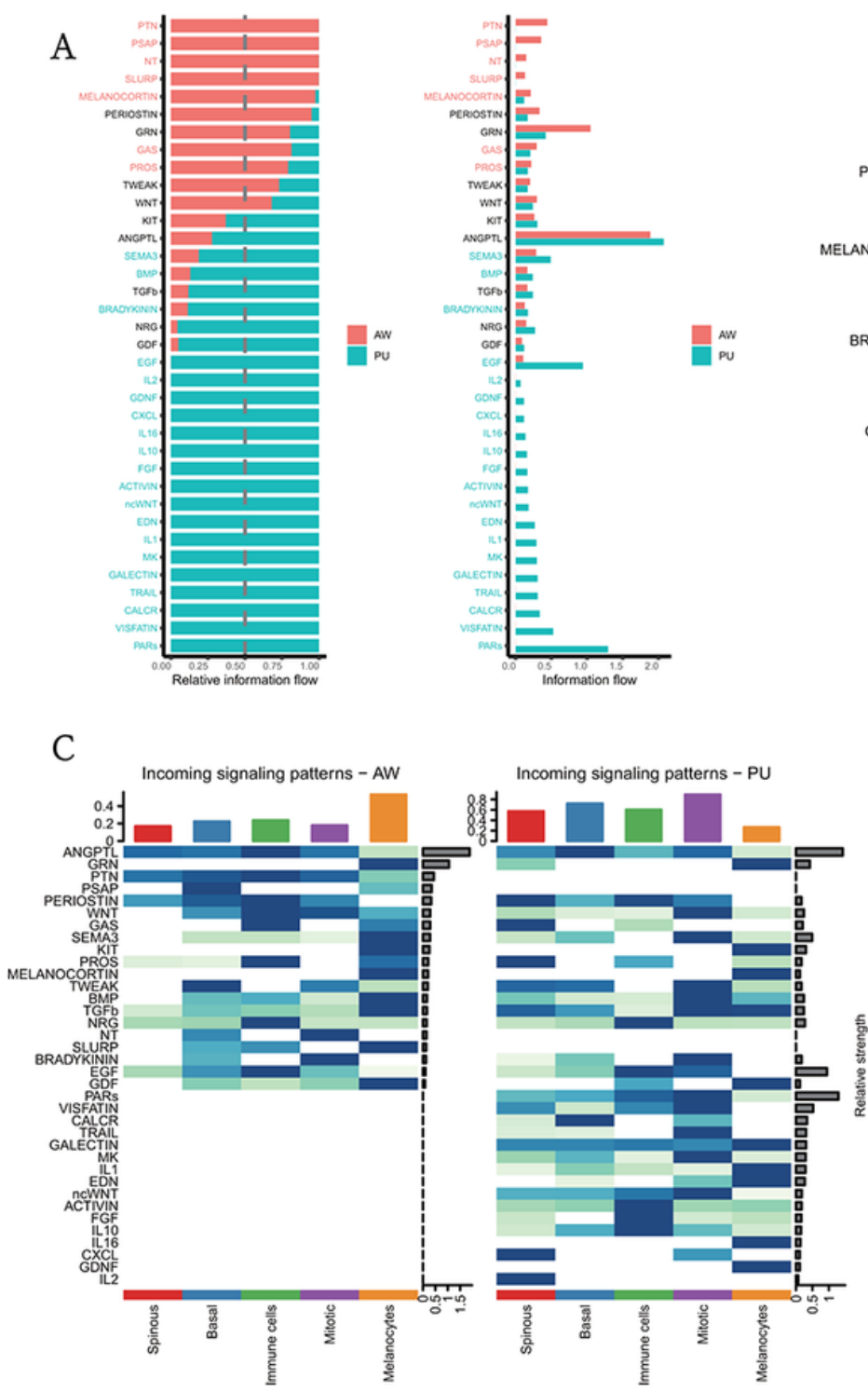
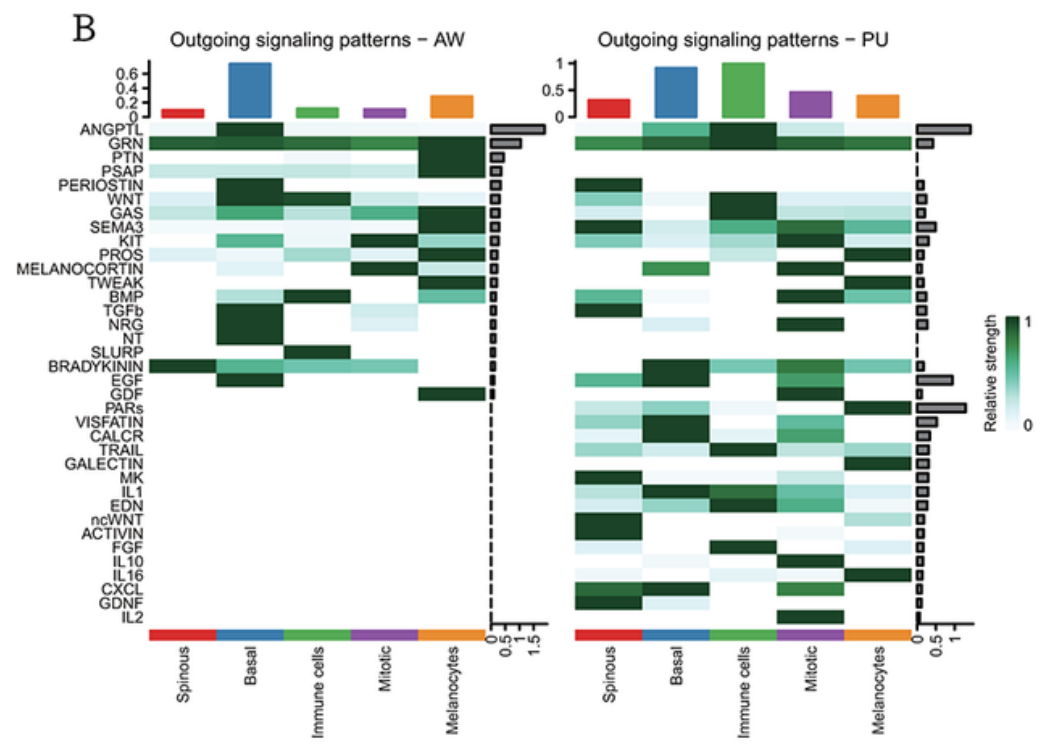

$\mathrm{D}$

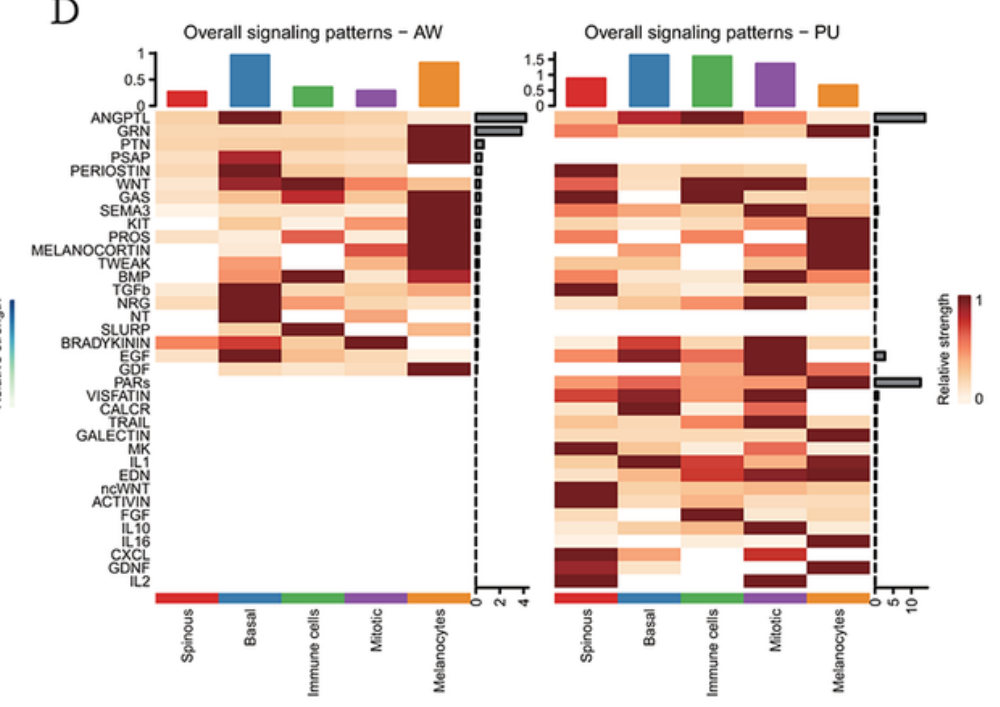

Figure 3

Comparison of the overall information flow of each signaling pathway. A. Significant signaling pathways were ranked based on differences in the overall information flow within the inferred networks between AW and PU. The signaling pathways depicted in red are enriched in AW, and those depicted in green were enriched in the PU. B, C, D Outgoing signals, incoming signals, and overall signaling by aggregating outgoing and incoming signaling together. 
A

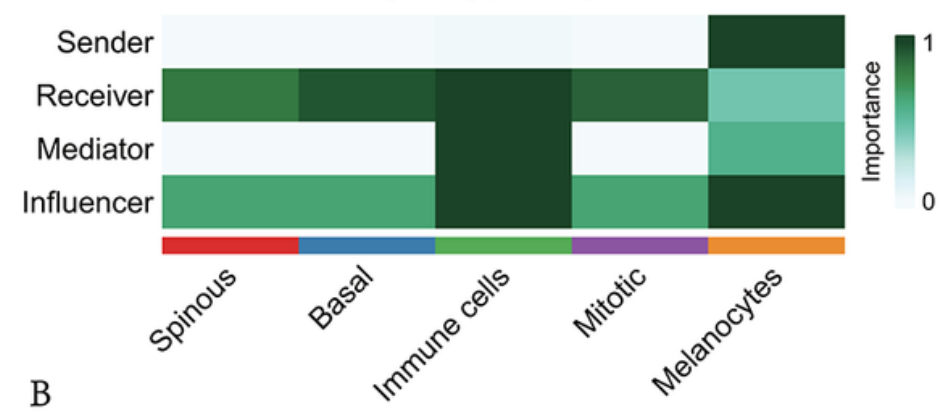

\section{PARs signaling pathway network}

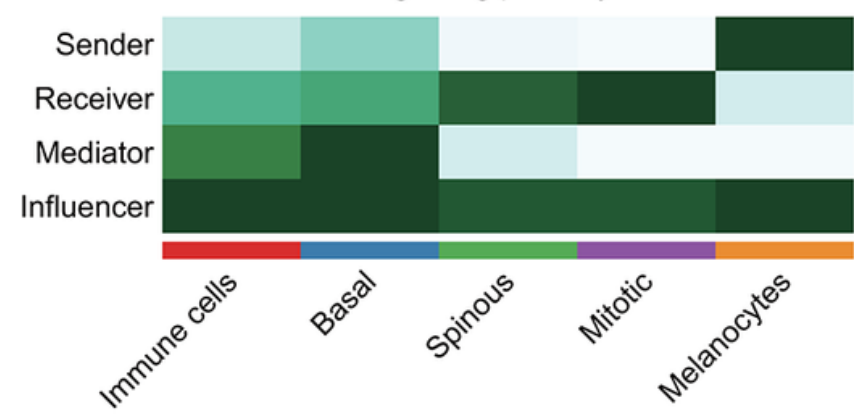

C

C Increased signaling in Pu

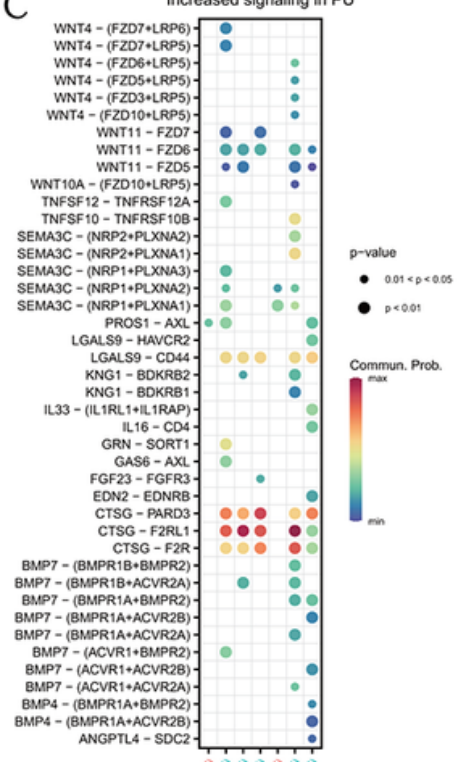

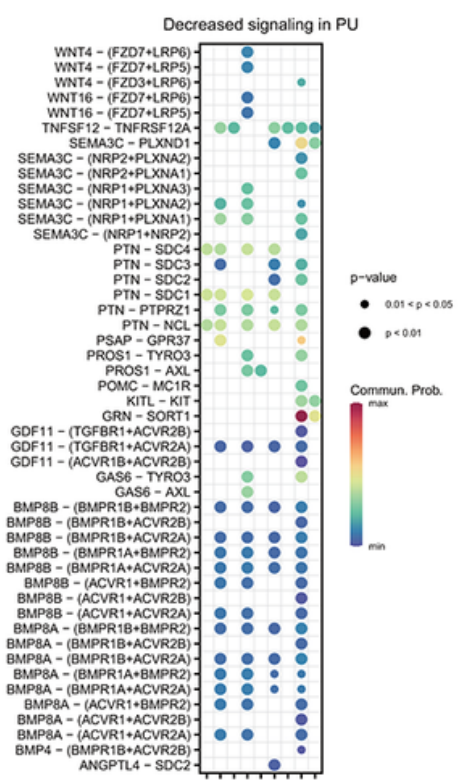

Figure 4

Identification of dominant senders, receivers, mediators, and influencers and determination of ligandreceptor pair communication strength in PTN and PAR signaling pathways. A, Dominant senders, receivers, mediators, and influencers of PTN signaling pathways. B, Dominant senders, receivers, mediators, and influencers of PARs signaling pathways. C, The upregulated (increased) and downregulated (decreased) signaling ligand-receptor pairs in PU compared to AW. The increased signaling means these pathways have a higher communication probability (strength) in PU compared to AW. 
A
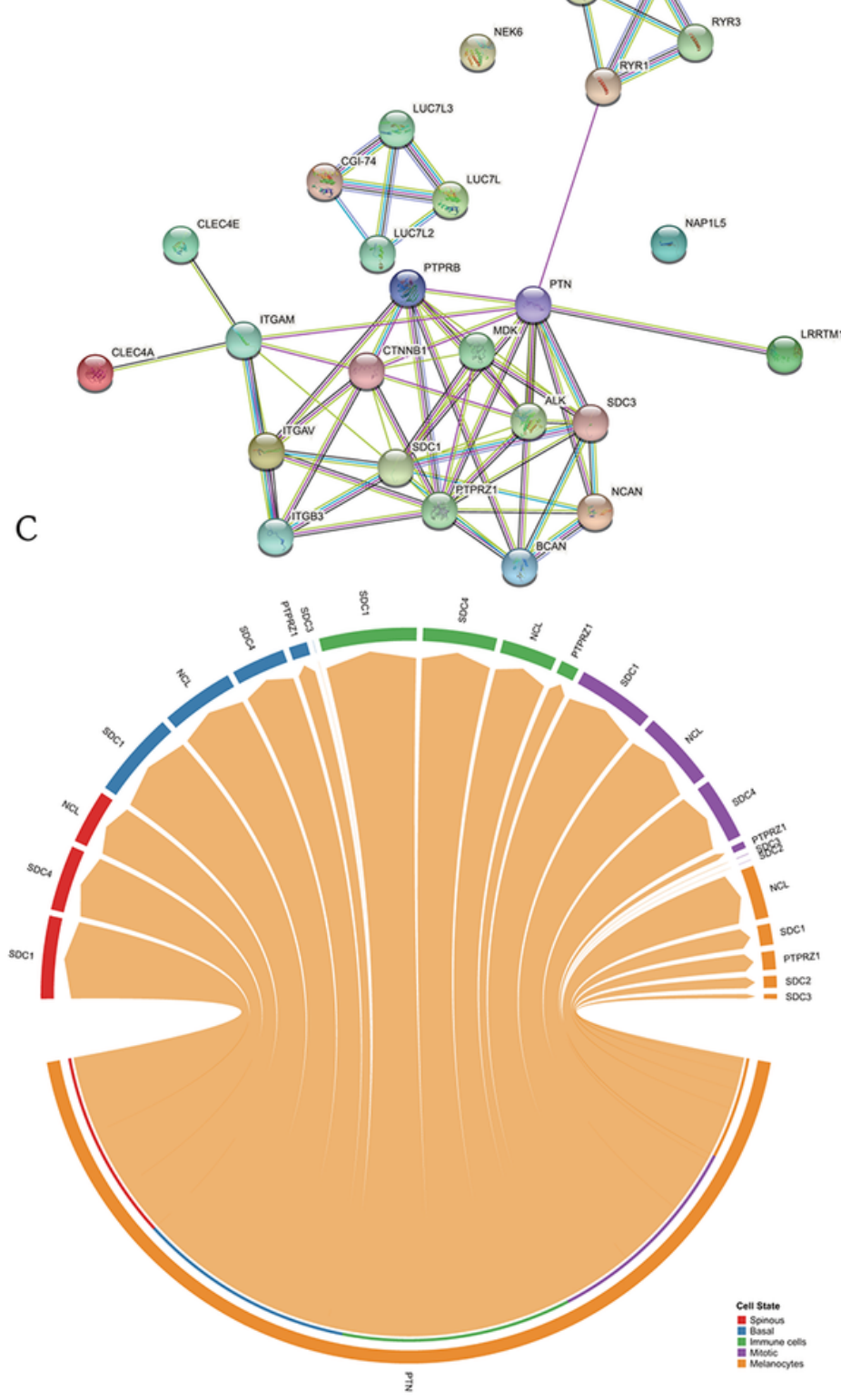

B

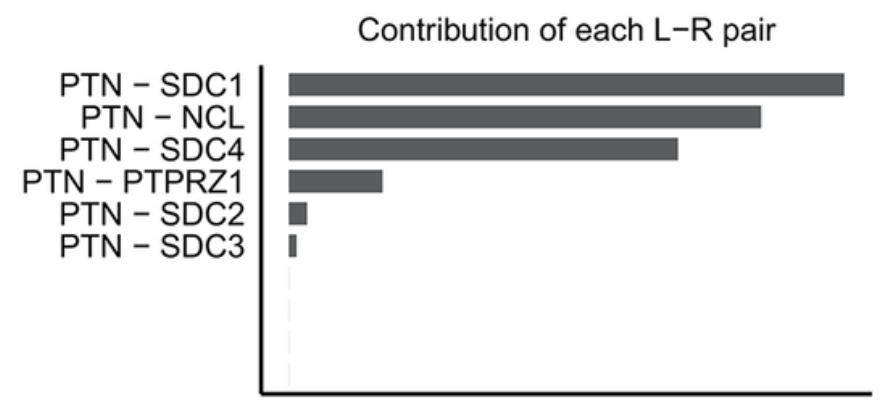

Relative contribution

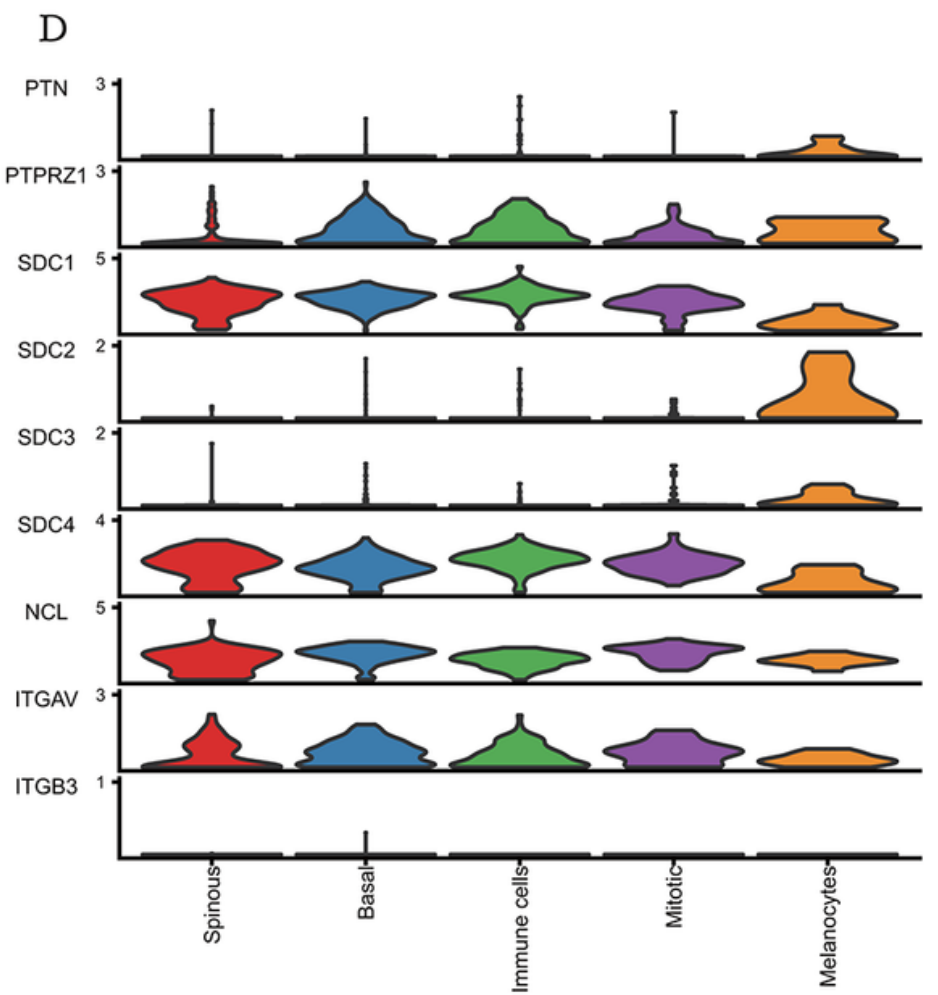

Figure 5

Effects of the PTN signaling pathway on the receiver cell population. A, STRING interaction network of PTN gene. B, The contribution of each ligand-receptor pair in the PTN pathway. C, The ligand-receptor pairs of PTN pathway from melanocytes population to the other cell populations in cell-cell communication. D, The gene expression distribution of signaling genes related to ligand-receptor pairs of PTN pathway. 


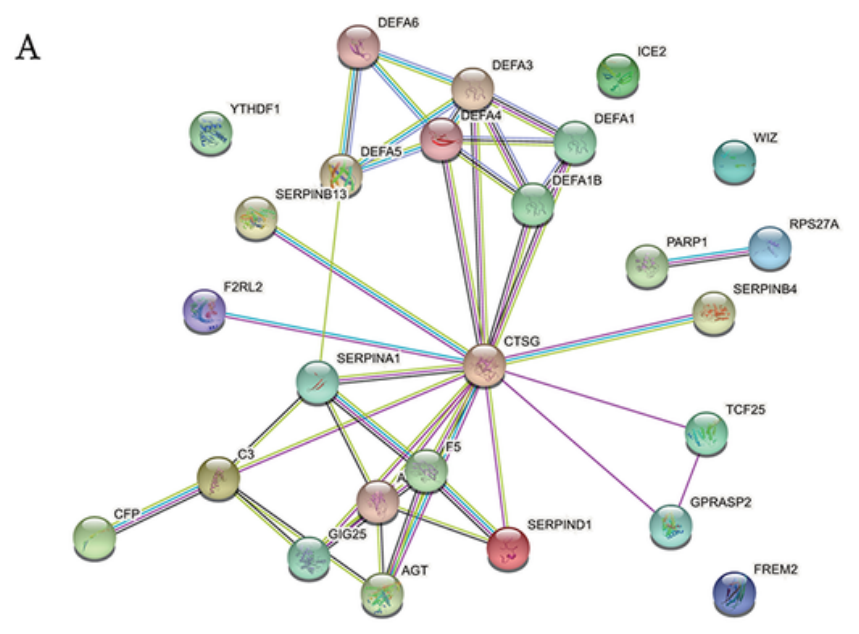

C

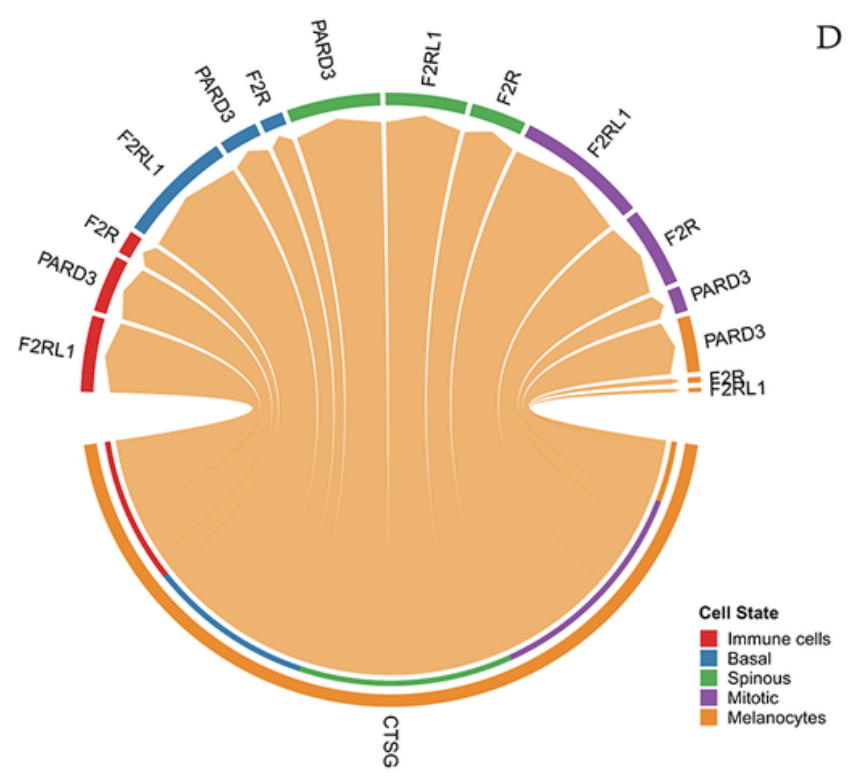

Contribution of each L-R pair

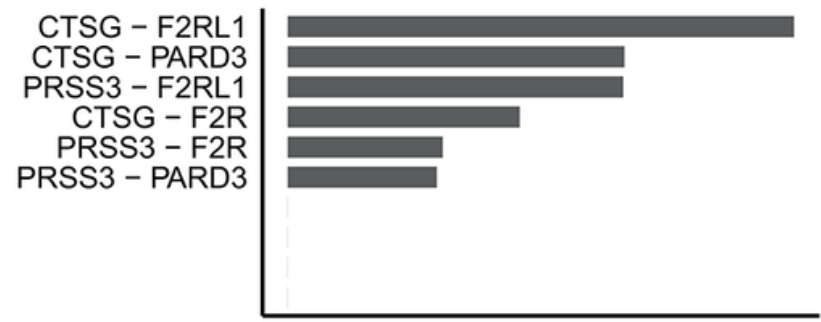

Relative contribution

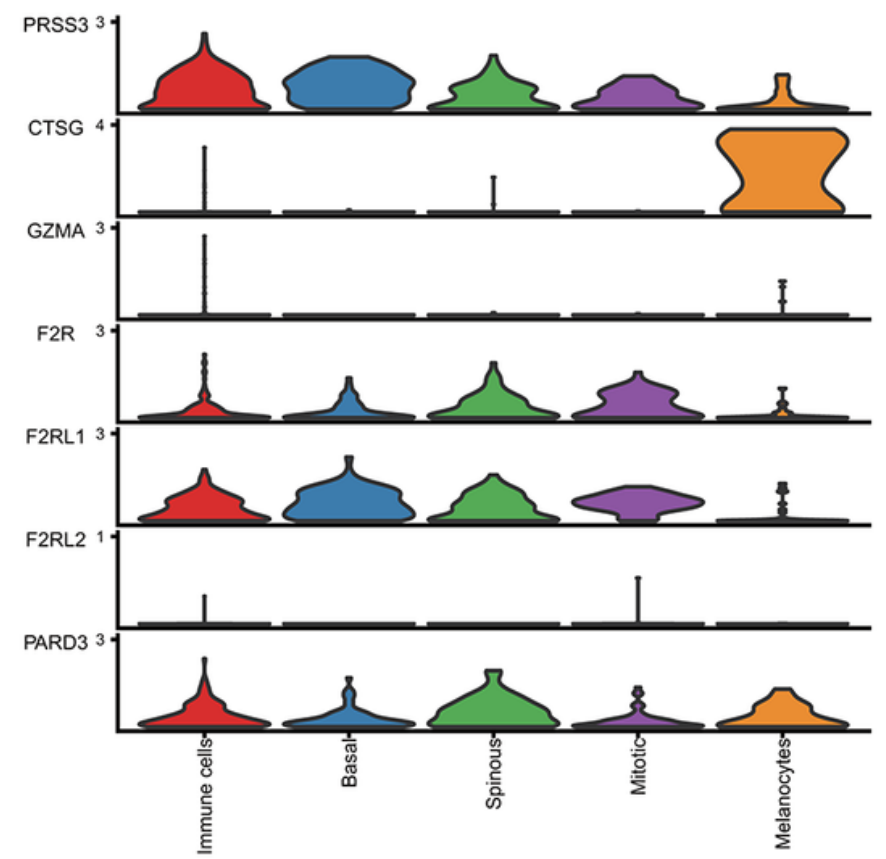

Figure 6

Effects of the PAR signaling pathway on the receiver cell population. A, STRING interaction network of PARs gene. B, The contribution of each ligand-receptor pair in the PAR pathway. C, The ligand-receptor pairs of PAR pathway from melanocytes population to the other cell populations in cell-cell communication. $\mathrm{D}$, The gene expression distribution of signaling genes related to ligand-receptor pairs of PARs pathway. 